\title{
AN HEREDITARY ENZYMATIC DEFECT IN ERYTHROCYTE METABOLISM: GLUCOSE-6-PHOSPHATE DEHYDROGENASE DEFICIENCY ${ }^{1}$
}

\author{
By RUTH T. GROSS, RUTH E. HURWITZ, AND PAUL A. MARKS \\ (From the Department of Pediatrics, Stanford University School of Medicine, \\ San Francisco, Calif., and Department of Medicine, College of Physicians \\ and Surgeons, Columbia University, New York, N. Y.)
}

(Submitted for publication January 10, 1958; accepted April 25, 1958)

Alterations in glutathione metabolism have been detected in the erythrocytes of patients with hemolytic anemia induced by ingestion of a variety of chemical agents including primaquine (1), acetanilid, sulfanilamide (2), naphthalene (3), fava beans (4) and nitrofurantoin (5). Erythrocytes sensitive to these agents may be identified by an in vitro test in which incubation with acetylphenylhydrazine leads to loss of reduced glutathione. Employing this test, it was found that among individuals without a previous history of hemolytic anemia, the incidence of subjects with drug sensitive erythrocytes was approximately 4.8 per cent in Negroes and apparently rare in Caucasians (6). The incidence and racial distribution of this defect in glutathione metabolism was similar to the previously reported distribution of primaquine sensitivity (7).

The reactions involved in the maintenance of glutathione in the reduced state may be illustrated by the following scheme ${ }^{2}(8-10)$ :
Carson, Flanagan, Ickes, and Alving (10) have demonstrated that the addition of 6-PG., but not G-6-P, to hemolysates of primaquine sensitive erythrocytes could initiate the above reactions leading to GSH formation. These authors interpreted their findings as indicating that the basic abno $\bar{r}$ mality in red blood cells of primaquine sensitive subjects was a deficiency in G-6-P.D. Recently, Waller, Löhr, and Tabatabai (11) reported an absence of G-6-P.D. and an accumulation of TPN in the erythrocytes of one patient with chronic hemolytic anemia of undetermined etiology.

In the present study a reduction in erythrocyte G-6-P.D. has been demonstrated in healthy subjects without a history of hemolytic disease as well as in patients who have had a drug induced hemolytic anemia. This investigation establishes that this enzymatic defect in erythrocyte metabolism is on an hereditary basis.
1. G-6-P $+\mathrm{TPN}^{+} \stackrel{\text { G-6-P.D. }}{\rightleftharpoons}$ 6-phosphogluconolactone $+\mathrm{TPNH}+\mathrm{H}^{+}$
2. 6-phosphogluconolactone $\stackrel{\text { Lactonase }}{\longrightarrow} 6-\mathrm{PG}$.
3. 6-PG. $+\mathrm{TPN}^{+} \stackrel{\text { 6-PG.D. }}{\rightleftharpoons} \mathrm{R}-5-\mathrm{P}+\mathrm{CO}_{2}+\mathrm{TPNH}+\mathrm{H}^{+}$
4. $\mathrm{GSSG}+\mathrm{TPNH}+\mathrm{H}^{+} \stackrel{\text { GSSG red. }}{\longrightarrow} 2 \mathrm{GSH}+\mathrm{TPN}^{+}$

\footnotetext{
1 This work was supported in part by Grant No. RG4754 of the United States Public Health Service, and Grant No. C-2332 of the National Cancer Institute, United States Public Health Service.

2 The following abbreviations are used: G-6-P., glucose6-phosphate; G-6-P.D., glucose-6-phosphate dehydrogenase; TPN, triphosphopyridine nucleotide; 6-PG., 6phosphogluconate; 6-PG.D.,-6-phosphogluconic dehydrogenase; TPNH, reduced triphosphopyridine nucleotide; R-5-P, ribulose-5-phosphate; GSSG, oxidized glutathione; GSH, reduced glutathione; GSSG red., glutathione reductase; PNP, purine nucleoside phosphorylase.
}

\section{METHODS}

Subjects studied. 1. Control subjects (Table I). The control subjects were selected from a group of individuals in good health and without a history of known hematological disease. Among 227 subjects tested, 13 were found to have a deficiency of G-6-P.D. and were excluded. The controls, those without deficiency of G-6-P.D., then comprised a group of 214 subjects, 98 Negroes and 116 Caucasians. Fifty-six of this group were newborn infants (20 Caucasians and 36 Negroes).

2. Subjects without known hematological disorder but zerith $a$ deficiency of erythrocyte G-6-P.D. This group 
included the 13 subjects discovered among the 227 healthy individuals tested (Table II). Four were Negro infants, 7 Negro adults, and 2 Caucasian adults. Family studies were obtained in 5 of these subjects : K. R.-father, mother and one sibling; W. C.-father, mother and five siblings; Baby F.-mother and father; M. H.-husband and four children; M. A.-one child. All the members of the families were in good health and none gave a history of hemolytic anemia.

3. Family studies of subjects with a history of naphthalene induced hemolytic anemia (Table III). Four weeks prior to study, Subject R. A. had hemolytic anemia with a history of moth ball ingestion. Family studies of this individual included the mother and father, one male and two female siblings. All members of this family were well at the time they were studied, except the mother who was hospitalized because of an incomplete abortion associated with an anemia secondary to blood loss. Subject $\mathrm{Mi}$. P. was studied one year following an acute episode of naphthalene induced hemolytic anemia. Family studies included the mother, father, one female and two male siblings, all of whom were well. R. B. was a subject who, two years prior to study, had an acute episode of naphthalene induced hemolytic anemia. Family studies included the mother, one female sibling and one male sibling, all of whom were well. Subject Do. C. had a history of naphthalene induced hemolytic anemia two years prior to study. Family studies included the maternal grandmother, paternal aunt, one female sibling, one male nonidentical twin, two male maternal half-siblings, and one female maternal half-sibling, all of whom were well. I. O. T. experienced naphthalene induced hemolytic anemia six years prior to study. Family studies included the mother, one female sibling and one male maternal half-sibling, none of whom had a history of hemolytic anemia.

4. Family studies of subjects with fava bean induced hemolytic anemia (Table IV). J. P. had a history of favism eight years prior to study. Family studies included the mother and one female maternal half-sibling, both of whom were well. Subject L. C. gave a history of favism eight years prior to this study. Family observations included the father (German) and mother (mixed European extraction) who were both well. D. D., an Italian female, had a history of favism five years ago. Family studies included her husband, two sons and one married daughter. The daughter's husband and two children, one male and one female, were also included. None of the family members gave a history of hemolytic anemia. K. K., a 12 year old female of Greek extraction, experienced favism seven years prior to the present workup. In her family, in which three generations were available for study, it was possible to test 37 blood relatives and 8 spouses of these relatives. Her father was dead as a result of a coronary occlusion and was not known to have had hemolytic anemia. In her immediate family, her mother, one male and three female siblings, one brotherin-law, one niece and one nephew were all well and were studied. Her mother had two male and two female
TABLE I

\begin{tabular}{|c|c|c|c|c|}
\hline $\begin{array}{r}\text { Glucose-6-phosp } \\
\text { gluconic dehy } \\
\text { phosphor } \\
(G S H\end{array}$ & $\begin{array}{l}\text { te dehydr } \\
\text { rogenase } \\
\text { ase ( } P N F \\
\text { in erythro }\end{array}$ & $\begin{array}{l}\text { genase } \\
\text { O-PG.D } \\
\text { and re } \\
\text { cytes of }\end{array}$ & $\begin{array}{l}\text { p-6-I } \\
\text { pur } \\
\text { ced } \\
\text { trol }\end{array}$ & $\begin{array}{l}\text { 6-phospho- } \\
\text { iucleoside } \\
\text { hione } \\
\text { ts }\end{array}$ \\
\hline & & dults & & \\
\hline Assay & $\begin{array}{l}\text { Number of } \\
\text { subjects }\end{array}$ & $\begin{array}{l}\text { Cauca- } \\
\text { sians }\end{array}$ & Negro & Mean \pm S.D. \\
\hline $\begin{array}{l}\text { G-6-P.D.* } \\
\text { 6-PG.D.* } \\
\text { PNP* }\end{array}$ & $\begin{array}{r}158 \\
78 \\
49\end{array}$ & $\begin{array}{l}96 \\
44 \\
26\end{array}$ & $\begin{array}{l}62 \\
34 \\
23\end{array}$ & $\begin{array}{r}16.2 \pm 2.2 \\
17.0 \pm 3.8 \\
145.0 \pm 20.8\end{array}$ \\
\hline $\begin{array}{l}\% \text { GSH } \\
\text { protected }\end{array}$ & 16 & 13 & 3 & $89.0 \pm 10.0$ \\
\hline & Neon & tal perio & & \\
\hline $\begin{array}{l}\text { G-6-P.D.* } \\
\text { 6-PG.D.* } \\
\text { \% GSH }\end{array}$ & $\begin{array}{l}56 \\
22\end{array}$ & $\begin{array}{r}20 \\
7\end{array}$ & $\begin{array}{l}36 \\
15\end{array}$ & $\begin{array}{l}20.4 \pm 3.3 \\
21.8 \pm 3.7\end{array}$ \\
\hline $\begin{array}{l}\text { protected } \\
0-55 \text { hrs. old } \\
\% \text { GSH }\end{array}$ & 20 & 7 & 13 & $32.0 \pm 10.0$ \\
\hline $\begin{array}{l}\text { protected } \\
55-92 \mathrm{hrs} \text {. old }\end{array}$ & 8 & 3 & 5 & $73.0 \pm 7.1$ \\
\hline
\end{tabular}

* Enzyme units are expressed as $\Delta O . D$. per minute per $\mathrm{Gm}$. hemoglobin.

$\dagger$ Neonatal period includes full term infants of one week of age and younger.

siblings, all of whom denied having had hemolytic anemia. One uncle, unmarried, was studied. The other uncle refused study but his wife and three male and two female children were tested. All were well. One aunt and her male child were studied, the latter had a history of hemolytic anemia induced by sulfanilamide administration 20 years previously. The other aunt, her husband, and five male and two female children were studied. Four of the children were married. Their spouses and their 12 children were studied. All were well.

5. Family studies of subjects with hemolytic anemia of unknown etiology (Table V). Baby T. had a mild hemolytic anemia with no evidence of blood group or $\mathrm{Rh}$ incompatability. Family studies included mother, father and two female siblings who were all well. Subject H. W. had an acute episode of hemolytic anemia of undetermined etiology two and one-half months previously. Family studies included the father, mother and one male sibling who were well.

Procedures. The techniques employed for the assay of erythrocyte enzyme activity have been previously described (12). Five ml. of heparinized venous blood was centrifuged for 10 minutes at $1,500 \times \mathrm{G}$. The plasma and buffy coat were removed and the erythrocytes washed twice in an isotonic potassium chloride solution buffered at $\mathrm{pH}$ 7.4. The red blood cells were resuspended in approximately two volumes of this potassium chloride solution. The preparation of erythrocytes was performed at $0^{\circ} \mathrm{C}$. Aliquots of the resuspended cells were lysed by freezing and thawing twice. Assays for G-6-P.D., 6PG.D. and P.N.P. were performed on these hemolysates employing methods (13-15) with modifications that have been described elsewhere (12). All enzyme activities 
TABLE II

Studies of individuals with deficient erythrocyte glucose-6-phosphate dehydrogenase, without known hematological disorders, and their families

\begin{tabular}{|c|c|c|c|c|c|c|c|c|c|}
\hline Subject & Age* & Sex & Race & G-6-P.D. & 6-PG.D. & PNP & $\underset{\text { GSH }}{\text { Initial }}$ & $\underset{\text { protected }}{\text { GSH }}$ & Hgb. \\
\hline Baby C. L. & 30 days & $\mathbf{M}$ & $\mathrm{N}$ & $\begin{array}{c}\Delta O . D . / \min \\
2.5\end{array}$ & $. / G m . H g b$. & & $m g . / G m . H g b$. & $\%$ & $\begin{array}{c}\mathrm{Gm} . / 100 \mathrm{ml} \\
12.5\end{array}$ \\
\hline $\begin{array}{l}\text { K. R. } \\
\text { Mother R. } \\
\text { Father R. } \\
\text { Brother R. }\end{array}$ & $\begin{array}{l}8 \text { mos. } \\
9 \text { mos. } \\
31 \text { yrs. } \\
35 \text { yrs. } \\
10 \text { yrs. }\end{array}$ & $\begin{array}{l}\mathbf{M} \\
\mathbf{F} \\
\mathbf{M} \\
\mathbf{M}\end{array}$ & $\begin{array}{l}\mathrm{N} \\
\mathrm{N} \\
\mathrm{N} \\
\mathrm{N}\end{array}$ & $\begin{array}{r}2.3 \\
2.3 \\
7.8 \\
1.7 \\
20.7\end{array}$ & $\begin{array}{l}10.8 \\
12.3 \\
13.7 \\
12.8 \\
20.0\end{array}$ & & $\begin{array}{l}0.6 \\
1.2 \\
1.0 \\
1.6\end{array}$ & $\begin{array}{l}28 \\
47 \\
15\end{array}$ & $\begin{array}{l}11.5 \\
12.6 \\
15.5 \\
10.8\end{array}$ \\
\hline $\begin{array}{l}\text { W. C. } \\
\text { Mother C. } \\
\text { Father C. } \\
\text { Sister C. } \\
\text { Sister C. } \\
\text { Sister C. } \\
\text { Brother C. } \\
\text { Brother C. }\end{array}$ & $\begin{array}{r}14 \text { mos. } \\
29 \text { yrs. } \\
31 \text { yrs. } \\
11 \text { yrs. } \\
10 \text { yrs. } \\
9 \text { yrs. } \\
6 \text { yrs. } \\
4 \frac{1}{2} \text { yrs. }\end{array}$ & $\begin{array}{l}M \\
F \\
M \\
F \\
F \\
F \\
M \\
M\end{array}$ & $\begin{array}{l}N \\
N \\
N \\
N \\
N \\
N \\
N \\
N\end{array}$ & $\begin{array}{r}5.7 \\
9.5 \\
11.3 \\
11.8 \\
7.0 \\
12.0 \\
2.7 \\
13.8\end{array}$ & $\begin{array}{r}24.2 \\
15.2 \\
14.1 \\
13.2 \\
10.7 \\
14.0 \\
9.6 \\
15.8\end{array}$ & & $\begin{array}{l}1.6 \\
1.1 \\
1.6 \\
1.4 \\
1.2 \\
1.6 \\
0.8 \\
1.6\end{array}$ & $\begin{array}{l}22 \\
75 \\
93 \\
80 \\
66 \\
95 \\
13 \\
84\end{array}$ & $\begin{array}{r}9.3 \\
12.9 \\
14.6 \\
13.8 \\
13.8 \\
14.0 \\
12.1 \\
11.1\end{array}$ \\
\hline $\begin{array}{l}\text { Baby F. } \\
\text { Mother F. } \\
\text { Father F. }\end{array}$ & $\begin{array}{l}2 \text { days } \\
4 \text { days } \\
22 \text { yrs. } \\
21 \text { yrs. }\end{array}$ & $\begin{array}{l}F \\
F \\
M\end{array}$ & $\begin{array}{l}\mathrm{N} \\
\mathrm{N} \\
\mathrm{N}\end{array}$ & $\begin{array}{r}8.3 \\
8.9 \\
15.0 \\
0.7\end{array}$ & $\begin{array}{r}12.2 \\
13.9 \\
18.2 \\
9.3\end{array}$ & & $\begin{array}{l}2.1 \\
1.7 \\
1.3 \\
0.6\end{array}$ & $\begin{array}{r}25 \\
57 \\
100 \\
41\end{array}$ & $\begin{array}{l}16.7 \\
16.4 \\
12.0 \\
14.6\end{array}$ \\
\hline $\begin{array}{l}\text { Mother H. } \\
\text { Father H. } \\
\text { Son H. } \\
\text { Daughter H. } \\
\text { Son H. } \\
\text { Son H. }\end{array}$ & $\begin{array}{l}36 \mathrm{yrs} . \\
35 \mathrm{yrs} . \\
11 \mathrm{yrs} \\
10 \mathrm{yrs} \\
13 \mathrm{yrs} \\
14 \mathrm{yrs} .\end{array}$ & $\begin{array}{l}\mathbf{F} \\
\mathbf{M} \\
\mathbf{M} \\
\mathbf{F} \\
\mathbf{M} \\
\mathbf{M}\end{array}$ & $\begin{array}{l}N \\
N \\
N \\
N \\
N \\
N\end{array}$ & $\begin{array}{r}7.4 \\
12.3 \\
12.3 \\
12.8 \\
2.0 \\
13.2\end{array}$ & 10.0 & & $\begin{array}{l}1.2 \\
1.2 \\
1.4 \\
1.0 \\
0.6 \\
1.0\end{array}$ & $\begin{array}{l}72 \\
81 \\
67 \\
94 \\
12 \\
89\end{array}$ & $\begin{array}{l}13.8 \\
17.4 \\
13.4 \\
13.0 \\
12.7 \\
11.9\end{array}$ \\
\hline $\begin{array}{l}\text { Mother A. } \\
\text { Baby A. }\end{array}$ & $\begin{array}{r}33 \text { yrs. } \\
2 \text { days }\end{array}$ & $\begin{array}{l}\mathrm{F} \\
\mathrm{M}\end{array}$ & $\begin{array}{l}\mathrm{W} \\
\mathrm{W}\end{array}$ & $\begin{array}{r}7.2 \\
18.1\end{array}$ & 18.1 & & $\begin{array}{l}1.8 \\
1.9\end{array}$ & 72 & $\begin{array}{l}15.5 \\
18.2\end{array}$ \\
\hline $\begin{array}{l}\text { G. W. }{ }^{\dagger} \\
\text { P. P. }{ }^{-} \\
\text {C. McM. } \dagger \\
\text { M. W. } \dagger \\
\text { B. D. } \dagger \\
\text { A. T. } \dagger \\
\text { C. M. } \dagger\end{array}$ & $\begin{array}{l}26 \text { yrs. } \\
33 \text { yrs. } \\
30 \text { yrs. } \\
63 \text { yrs. } \\
70 \text { yrs. } \\
33 \text { yrs. } \\
70 \text { yrs. }\end{array}$ & $\begin{array}{l}\mathbf{F} \\
\mathbf{M} \\
\mathbf{M} \\
\mathbf{M} \\
\mathbf{M} \\
\mathbf{M} \\
\mathbf{F}\end{array}$ & $\begin{array}{l}\mathbf{N} \\
\mathbf{N} \\
\mathbf{N} \\
\mathbf{W} \\
\mathbf{N} \\
\mathbf{N} \\
\mathbf{N}\end{array}$ & $\begin{array}{l}7.4 \\
6.2 \\
4.4 \\
6.6 \\
3.4 \\
6.1 \\
6.6\end{array}$ & $\begin{array}{l}10.2 \\
12.3 \\
11.1 \\
16.0 \\
13.5 \\
12.4 \\
14.2\end{array}$ & $\begin{array}{l}140 \\
125 \\
131 \\
174 \\
150 \\
160\end{array}$ & 0.8 & & $\begin{array}{l}10.8 \\
16.7 \\
15.9 \\
13.5 \\
14.6 \\
12.3 \\
11.4\end{array}$ \\
\hline
\end{tabular}

* Age at time of study.

$\dagger$ Family studies not obtained.

were expressed as the change in optical density units per minute per gram of hemoglobin ( $\Delta$ O.D. per minute per Gm. Hgb.).

Reduced glutathione was measured by the method of Grunert and Phillips (16). The change in erythrocyte concentrations of GSH following a two hour incubation with acetylphenylhydrazine was employed as the basis for determining erythrocyte GSH stability according to the procedure of Beutler (6). Hemoglobin concentrations were measured by pipetting a suitable quantity of hemolysate into $10 \mathrm{ml}$. of 0.4 per cent $\mathrm{NH}_{4} \mathrm{OH}$ and reading the optical density of the resulting solution in a spectrophotometer at $540 \mathrm{~m} \mu$.

\section{RESULTS}

Table I summarizes the control data for erythrocyte concentrations of the enzymes, G-6-P.D., 6-
PG.D. and PNP and values of the GSH stability test. Tables II through $\mathrm{V}$ present the details of the studies performed on subjects with a deficiency in erythrocyte G-6-P.D. and their families.

\section{Glucose-6-phosphate dehydrogenase}

Decreased levels of erythrocyte G-6-P.D. were found in three categories of individuals: 1) 4 Negro infants discovered in the course of testing 135 infants ( 31 Negro and 54 Caucasian),$^{3}$ and

${ }^{3}$ Erythrocytes from a total of 135 infants were assayed for G-6-P.D. activity. Four of these had significantly low levels of enzyme, leaving a total control group of 131. In only 56 of these infants were hemoglobin assays performed on the hemolysates used for enzyme analysis 
TABLE III

Family studies of individuals with naphthalene hemolytic anemia

\begin{tabular}{|c|c|c|c|c|c|c|c|c|c|}
\hline Subject & Age* & Sex & Race & G-6-P.D. & 6-PG.D. & PNP & $\begin{array}{l}\text { Initial } \\
\text { GSH }\end{array}$ & $\underset{\text { protected }}{\text { GSH }}$ & Hgb. \\
\hline & & & & \multicolumn{2}{|c|}{$\Delta O . D . / \min . / G m . \mathrm{Hg} b$} & & \multirow{8}{*}{$\begin{array}{c}m g . / G m . H g b . \\
1.9 \\
1.7 \\
1.8 \\
1.5 \\
1.6 \\
1.3 \\
1.3 \\
0.8\end{array}$} & \multirow{8}{*}{$\begin{array}{r}\% \\
25 \\
7 \\
54 \\
56 \\
100 \\
17 \\
62 \\
92\end{array}$} & \multirow{8}{*}{$\begin{array}{c}G m . / 100 \mathrm{ml} . \\
11.3 \\
11.8 \\
8.2 \\
11.8 \\
16.5 \\
10.6 \\
12.4 \\
19.8\end{array}$} \\
\hline R. A. $\dagger$ & 14 mos. & $\mathrm{F}$ & $N$ & 4.1 & 20.7 & & & & \\
\hline & 15 mos. & & & 1.5 & 12.7 & & & & \\
\hline Mother A. & 24 yrs. & $\mathrm{F}$ & $\mathrm{N}$ & 9.1 & 15.6 & & & & \\
\hline Father A. & 25 vrs. & $\mathbf{M}$ & $\mathrm{N}$ & $\begin{array}{r}7.0 \\
13.7\end{array}$ & $\begin{array}{l}16.3 \\
14.7\end{array}$ & & & & \\
\hline Brother A. & 4 yrs. & $\mathbf{M}$ & $\mathrm{N}$ & 2.4 & 12.3 & & & & \\
\hline Sister A. & 6 yrs. & $\mathbf{F}$ & $\mathrm{N}$ & 8.0 & 13.2 & & & & \\
\hline Sister A. & 8 yrs. & $\mathbf{F}$ & $\mathrm{N}$ & 16.3 & 17.4 & & & & \\
\hline Mi. P.† & 3 yrs. & $\mathbf{M}$ & $\mathrm{N}$ & 4.5 & 13.3 & 152 & & & 13.3 \\
\hline Mother P. & 35 yrs. & $\mathrm{F}$ & $\mathbf{N}$ & 3.6 & 12.6 & 141 & & & 12.2 \\
\hline Father P. & 37 yrs. & $\mathbf{M}$ & $\mathrm{N}$ & 17.4 & 12.6 & 126 & & & 13.9 \\
\hline Brother $\mathrm{P}$. & 7 yrs. & $\mathbf{M}$ & $\mathrm{N}$ & 2.4 & 13.4 & 147 & & & 12.0 \\
\hline Brother P. & 5 yrs. & $\mathbf{M}$ & $\mathrm{N}$ & 2.5 & 13.6 & 131 & & & 12.5 \\
\hline Sister P. & 10 yrs. & $\mathrm{F}$ & $\mathrm{N}$ & 10.9 & 10.2 & 143 & & & 13.8 \\
\hline R. B.† & 3 yrs. & $\mathbf{M}$ & $\mathbf{N}$ & 3.4 & 13.0 & 159 & & & 13.4 \\
\hline Mother B. & 29 yrs. & $\mathbf{F}$ & $\mathbf{N}$ & 13.3 & 13.3 & 134 & & & 13.5 \\
\hline Sister B. & 4 yrs. & $F$ & $\mathbf{N}$ & 21.0 & 22.6 & 154 & & & \\
\hline Brother B. & $1 \mathrm{yr}$. & $\mathbf{M}$ & $\mathrm{N}$ & 5.0 & & 196 & & & 13.0 \\
\hline Do. C. $\dagger$ & 5 yrs. & $\mathbf{M}$ & $\mathbf{N}$ & 2.7 & 12.9 & 157 & & & 13.0 \\
\hline Grandmother & 63 yrs. & $\mathrm{F}$ & $\mathrm{N}$ & 16.0 & 18.9 & 105 & & & 13.6 \\
\hline Paternal aunt & 49 yrs. & $\mathbf{F}$ & $\mathbf{N}$ & 19.1 & 16.1 & 186 & & & 12.0 \\
\hline Sister C. & 8 yrs. & $\mathbf{F}$ & $\mathbf{N}$ & 6.6 & 18.1 & 147 & & & 13.9 \\
\hline Twin C. & 5 yrs. & M & $\mathbf{N}$ & 19.7 & 17.6 & 149 & & & 13.2 \\
\hline Half-brother & 4 yrs. & $\mathbf{M}$ & $\mathrm{N}$ & 17.8 & 16.7 & 131 & & & 13.6 \\
\hline Half-brother & 2 yrs. & $\mathbf{M}$ & $\mathrm{N}$ & 20.1 & 18.1 & 121 & & & 13.8 \\
\hline Half-sister & 3 yrs. & $\mathbf{M}$ & $\mathrm{N}$ & 17.3 & 17.2 & 131 & & & 13.4 \\
\hline I. O. T. $\dagger$ & 8 yrs. & M & $\mathrm{N}$ & 1.5 & & & 0.8 & 20 & 12.2 \\
\hline Mother $\mathrm{T}$. & 35 yrs. & $\mathbf{F}$ & $\mathrm{N}$ & 2.0 & & & 0.7 & 40 & 13.1 \\
\hline Half-brother $\mathrm{T}$. & 16 yrs. & $\mathbf{M}$ & $\mathrm{N}$ & 1.5 & 9.7 & & 0.7 & 28 & 16.1 \\
\hline Sister T. & 2 yrs. & $\mathbf{F}$ & $\mathrm{N}$ & 6.6 & 11.7 & & 0.8 & 61 & 13.8 \\
\hline
\end{tabular}

* Age at time of study.

$\dagger$ Propositus who had hemolytic anemia.

9 adults ( 7 Negro and 2 Caucasian) discovered in the course of testing 170 adults ( 71 Negro and 99 Caucasian), none of whom had known hematological disorders; 2) 8 Negro and 3 Caucasian individuals known to have had hemolytic anemia -5 following naphthalene ingestion, 4 after fava bean consumption, and 2 of undetermined etiology ; 3) 39 (26 Negro and 13 Caucasian) relatives of the above individuals. One Caucasian

so as to provide the necessary data to express the units of enzyme activity per gram of hemoglobin. In the other 75 infants, the enzyme concentrations were referred to a volume of packed erythrocytes and, therefore, could not be used to compare with the control adult data or the data on the subjects with G-6-P.D. deficiency. Accordingly, the control data as presented in Table I include only 56 infants. However, for a correct impression as to the incidence of the G-6-P.D. deficiency, the four infants with a deficiency in erythrocyte G-6-P.D. without known hematological disorder must be considered in relation to a total group of 135 . male in this group of relatives gave a history of sulfonamide induced hemolytic anemia. A total of 103 (49 Negro and 54 Caucasian) relatives were studied.

Among the 52 subjects with a decreased erythrocyte G-6-P.D. content but no history of hematological disease, 24 had moderate reductions in G-6-P.D. All values in this group were between 2.5 and 4.0 standard deviations from the control mean. The mean value for the intermediate group was $8.9 \Delta \mathrm{O}$. D. per minute per $\mathrm{Gm}$. Hgb. with a standard deviation of \pm 1.3 . Of these 24 individuals, 23 were females. The remaining 28 subjects, and 12 of the 13 with a history of hemolytic anemia, had levels of erythrocyte G-6-P.D. which fell below 4 standard deviations of the mean for the control subjects. The mean value for the group with low enzymes was $2.75 \Delta \mathrm{O}$. D. per minute per $\mathrm{Gm}$. Hgb., with a standard deviation of \pm 2.0 . 
TABLE IV

Family studies of individuals with favism

\begin{tabular}{|c|c|c|c|c|c|c|c|c|}
\hline Subject & Age* & Sex & Race & G-6-P.D. & 6-PG.D. & $\begin{array}{c}\text { Initial } \\
\text { GSH }\end{array}$ & $\underset{\text { protected }}{\text { GSH }}$ & Hgb. \\
\hline $\begin{array}{l}\text { J. P.† } \\
\text { Mother P. } \\
\text { Sister P. }\end{array}$ & $\begin{array}{r}y r s . \\
12 \\
21 \\
6\end{array}$ & $\begin{array}{l}\mathbf{M} \\
\mathbf{F} \\
\mathbf{F}\end{array}$ & $\begin{array}{l}\mathbf{N} \\
\mathbf{N} \\
\mathbf{N}\end{array}$ & $\begin{array}{c}\Delta O . D . / m i \\
1.9 \\
9.1 \\
2.4\end{array}$ & $\begin{array}{c}\text { /Gm. Hgb. } \\
11.3 \\
14.9 \\
9.8\end{array}$ & $\begin{array}{c}m g . / G m . H g b . \\
1.3\end{array}$ & $\begin{array}{c}\% \\
14\end{array}$ & $\begin{array}{c}G m . / 100 \mathrm{ml} . \\
12.9\end{array}$ \\
\hline $\begin{array}{l}\text { D. D.† } \\
\text { Husband B. D. } \\
\text { Son Do. D. } \\
\text { Son A. D. } \\
\text { Daughter A. L. } \\
\text { Husband L. } \\
\text { Son J. L. } \\
\text { Daughter E. L. }\end{array}$ & $\begin{array}{r}50 \\
64 \\
32 \\
22 \\
28 \\
30 \\
6 \\
4\end{array}$ & $\begin{array}{l}\mathbf{F} \\
\mathbf{M} \\
\mathbf{M} \\
\mathbf{M} \\
\mathbf{F} \\
\mathbf{M} \\
\mathbf{M} \\
\mathbf{F}\end{array}$ & $\begin{array}{l}\mathbf{W} \\
\mathbf{W} \\
\mathbf{W} \\
\mathbf{W} \\
\mathbf{W} \\
\mathbf{W} \\
\mathbf{W} \\
\mathbf{W}\end{array}$ & $\begin{array}{r}0.4 \\
12.6 \\
0.1 \\
0.1 \\
9.0 \\
14.9 \\
0.1 \\
9.0\end{array}$ & $\begin{array}{l}12.6 \\
15.2 \\
10.5 \\
12.0 \\
14.0 \\
10.9 \\
12.0 \\
16.0\end{array}$ & $\begin{array}{l}0.8 \\
0.9 \\
0.4 \\
0.4 \\
1.0 \\
\\
0.6 \\
1.1\end{array}$ & $\begin{array}{r}6 \\
98 \\
22 \\
30 \\
93 \\
\\
15 \\
74\end{array}$ & $\begin{array}{l}15.8 \\
17.0 \\
19.5 \\
15.8 \\
14.1 \\
15.0 \\
13.4 \\
13.2\end{array}$ \\
\hline $\begin{array}{l}\text { L. C. } \dagger \\
\text { Mother C. } \\
\text { Father C. }\end{array}$ & $\begin{array}{l}12 \\
21+ \\
21+\end{array}$ & $\begin{array}{l}\mathbf{M} \\
\mathbf{F} \\
\mathbf{M}\end{array}$ & $\begin{array}{l}\mathbf{W} \\
\mathbf{W} \\
\mathbf{W}\end{array}$ & $\begin{array}{r}0.4 \\
8.2 \\
16.5\end{array}$ & $\begin{array}{l}10.5 \\
14.0 \\
17.0\end{array}$ & $\begin{array}{l}0.6 \\
1.6 \\
1.3\end{array}$ & $\begin{array}{l}19 \\
75 \\
96\end{array}$ & $\begin{array}{l}15.2 \\
13.9 \\
15.9\end{array}$ \\
\hline $\begin{array}{l}\text { K. K.† } \\
\text { Mother K. } \\
\text { Brother T. K. } \\
\text { Sister N. K. } \\
\text { Sister A. K. } \\
\text { Sister A. H. } \\
\text { Husband R. H. } \\
\text { Daughter D. H. } \\
\text { Son B. H. } \\
\text { Maternal uncle W. S. } \\
\text { Maternal aunt M. D. } \\
\text { Son A. D.f } \\
\text { Maternal aunt A. S. } 8 \\
\text { Daughter A. S. } \\
\text { Daughter K. S. } \\
\text { Son T.S. } \\
\text { Son B. S. } \\
\text { Son E. S. } \\
\text { Maternal aunt E. K. } \\
\text { Husband A. K. } \\
\text { Son A. K. } \\
\text { Wife S. K. } \\
\text { Daughter L. K. } \\
\text { Son P. K. } \\
\text { Son J. K. } \\
\text { Son G. K. } \\
\text { Wife E. K. } \\
\text { Daughter V. K. } \\
\text { Daughter L. K. } \\
\text { Daughter Co. K. } \\
\text { Son N.K. } \\
\text { Son Go. K. } \\
\text { Wife P. K. } \\
\text { Son D. K. } \\
\text { Son M. K. } \\
\text { Daughter K. K. } \\
\text { Daughter Ki. K. } \\
\text { Daughter S. W. } \\
\text { Husband D. W. } \\
\text { Daughter D. W. } \\
\text { Daughter K. L. } \\
\text { Husband D. L. } \\
\text { Daughter V. L. } \\
\text { Son M. L. } \\
\text { Son L. L. } \\
\text { Dong }\end{array}$ & $\begin{array}{r}12 \\
50 \\
14 \\
21 \\
19 \\
23 \\
25 \\
2 \\
4 \\
40 \\
52 \\
25 \\
60 \\
20 \\
28 \\
25 \\
24 \\
27 \\
59 \\
60 \\
30 \\
22 \\
1 \\
21 \\
19 \\
26 \\
22 \\
6 \\
5 \\
1 \\
3 \\
24 \\
23 \\
2 \\
3 \\
6 \\
1 \\
29 \\
33 \\
1 \\
28 \\
33 \\
7 \\
5 \\
1\end{array}$ & $\begin{array}{l}\mathbf{F} \\
\mathbf{M} \\
\mathbf{F} \\
\mathbf{F} \\
\mathbf{M} \\
\mathbf{F} \\
\mathbf{M} \\
\mathbf{F} \\
\mathbf{M} \\
\mathbf{F} \\
\mathbf{F} \\
\mathbf{F} \\
\mathbf{M} \\
\mathbf{M} \\
\mathbf{F} \\
\mathbf{M} \\
\mathbf{M} \\
\mathbf{F} \\
\mathbf{F} \\
\mathbf{M} \\
\mathbf{M} \\
\mathbf{F} \\
\mathbf{F} \\
\mathbf{F} \\
\mathbf{F} \\
\mathbf{M} \\
\mathbf{M} \\
\mathbf{F} \\
\mathbf{M} \\
\mathbf{M} \\
\mathbf{F} \\
\mathbf{F} \\
\mathbf{M} \\
\mathbf{F} \\
\mathbf{M} \\
\mathbf{F} \\
\mathbf{M} \\
\mathbf{M}\end{array}$ & 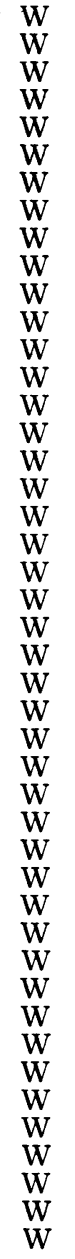 & $\begin{array}{r}7.8 \\
7.6 \\
15.0 \\
11.0 \\
14.1 \\
8.6 \\
12.6 \\
14.3 \\
11.7 \\
12.2 \\
8.7 \\
0.0 \\
10.9 \\
11.4 \\
11.8 \\
10.9 \\
10.7 \\
12.1 \\
0.0 \\
13.9 \\
0.0 \\
14.5 \\
11.5 \\
12.3 \\
11.3 \\
12.5 \\
16.0 \\
13.8 \\
13.5 \\
17.5 \\
16.7 \\
11.7 \\
12.5 \\
19.5 \\
14.2 \\
15.3 \\
16.6 \\
14.7 \\
13.5 \\
19.9 \\
11.3 \\
13.1 \\
15.3 \\
13.6 \\
16.0\end{array}$ & $\begin{array}{r}7.6 \\
10.0 \\
10.0 \\
11.0 \\
14.7 \\
16.3 \\
10.0 \\
15.6 \\
12.9 \\
9.8 \\
9.9 \\
11.7\end{array}$ & $\begin{array}{l}1.0 \\
1.3 \\
1.1 \\
0.7 \\
1.1 \\
0.8 \\
1.4 \\
0.9 \\
0.9 \\
0.9 \\
1.3 \\
0.6 \\
1.3 \\
1.4 \\
1.5 \\
1.2 \\
0.9 \\
0.6 \\
0.8 \\
1.7 \\
0.9 \\
1.5 \\
1.6 \\
1.6 \\
1.1 \\
1.6 \\
1.6 \\
1.3 \\
1.4 \\
1.0 \\
11.6 \\
1.9 \\
1.9 \\
1.4 \\
1.2 \\
2.0 \\
1.6 \\
1.5 \\
1.6 \\
1.5 \\
1.3 \\
1.3 \\
1.4 \\
1.8\end{array}$ & $\begin{array}{r}100 \\
100 \\
100 \\
91 \\
34 \\
86 \\
95 \\
95 \\
98 \\
93 \\
47 \\
23 \\
100 \\
13 \\
83 \\
67 \\
88 \\
\\
81 \\
89 \\
89 \\
100 \\
100 \\
75 \\
94 \\
\\
\\
100 \\
90 \\
100 \\
100 \\
100 \\
91 \\
88 \\
88 \\
90\end{array}$ & $\begin{array}{l}14.8 \\
13.6 \\
16.7 \\
14.0 \\
13.8 \\
14.1 \\
15.0 \\
12.2 \\
13.9 \\
16.0 \\
12.5 \\
12.5 \\
14.4 \\
13.9 \\
13.7 \\
16.6 \\
16.7 \\
16.5 \\
13.8 \\
12.6 \\
15.3 \\
13.2 \\
12.8 \\
16.8 \\
15.7 \\
16.8 \\
14.0 \\
14.3 \\
13.0 \\
11.0 \\
12.7 \\
15.7 \\
12.9 \\
11.8 \\
11.8 \\
12.4 \\
10.6 \\
16.3 \\
18.0 \\
11.9 \\
13.9 \\
15.9 \\
12.5 \\
12.0 \\
12.0\end{array}$ \\
\hline
\end{tabular}

* Age at time of study.

$\dagger$ Propositus who had hemolytic anemia.

History of sulfonamide induced hemolytic anemia 20 years ago.

Related by marriage. 
TABLE V

Family studies of individuals with hemolytic anemia of unknown cause

\begin{tabular}{|c|c|c|c|c|c|c|c|c|}
\hline Subject & Age* & Sex & Race & G-6-P.D. & 6-PG.D. & $\begin{array}{c}\text { Initial } \\
\text { GSH }\end{array}$ & $\underset{\text { protected }}{\text { GSH }}$ & Hgb. \\
\hline & & & & \multicolumn{2}{|c|}{$\Delta O . D . / \min . / G m . H g b}$. & mg./Gm. Hgb. & $\%$ & $\mathrm{Gm} . / 100 \mathrm{ml}$. \\
\hline Baby T.† & $\begin{array}{l}1 \text { day } \\
2 \text { days } \\
3 \text { days } \\
6 \text { days }\end{array}$ & $\mathbf{M}$ & $\mathbf{N}$ & $\begin{array}{l}4.8 \\
4.5 \\
4.4 \\
3.1\end{array}$ & $\begin{array}{l}15.3 \\
15.4 \\
14.0 \\
13.6\end{array}$ & $\begin{array}{l}0.1 \\
1.2 \\
1.3 \\
1.4\end{array}$ & $\begin{array}{r}0 \\
16 \\
12\end{array}$ & $\begin{array}{l}14.5 \\
14.7 \\
13.4 \\
12.7\end{array}$ \\
\hline Father $T$. & 33 yrs. & $\mathbf{M}$ & $\mathrm{N}$ & 10.7 & 12.8 & 1.8 & 75 & 15.4 \\
\hline Mother $T$. & 31 yrs. & $\mathbf{F}$ & $\mathrm{N}$ & 10.9 & 16.1 & 1.3 & 55 & 14.7 \\
\hline Sister T. & 5 yrs. & $\mathbf{F}$ & $\mathrm{N}$ & 10.5 & 13.1 & 1.5 & 63 & 12.2 \\
\hline Sister T. & $3 \frac{1}{2}$ yrs. & $\mathrm{F}$ & $\mathrm{N}$ & 14.5 & 14.5 & 1.4 & 92 & 12.9 \\
\hline $\begin{array}{l}\text { H. W. } \dagger \\
\text { Father W. } \\
\text { Brother W. } \\
\text { Mother W. }\end{array}$ & $\begin{array}{l}8 \text { yrs. } \\
40 \text { yrs. } \\
11 \text { yrs. } \\
38 \text { yrs. }\end{array}$ & $\begin{array}{l}\mathbf{M} \\
\mathbf{M} \\
\mathbf{M} \\
\mathbf{F}\end{array}$ & $\begin{array}{l}\mathbf{N} \\
\mathbf{N} \\
\mathbf{N}\end{array}$ & $\begin{array}{l}2.3 \\
0.7 \\
1.5 \\
6.7\end{array}$ & $\begin{array}{r}11.3 \\
8.4 \\
11.2 \\
10.0\end{array}$ & $\begin{array}{l}0.8 \\
0.8 \\
0.5 \\
1.2\end{array}$ & $\begin{array}{l}34 \\
45 \\
45 \\
67\end{array}$ & $\begin{array}{l}11.8 \\
15.4 \\
12.0 \\
12.8\end{array}$ \\
\hline
\end{tabular}

* Age at time of study.

$\dagger$ Propositus who had hemolytic anemia.

\section{Glutathione stability}

The control values for per cent of GSH protected after incubation with acetylphenylhydrazine are shown in Table I. Erythrocytes with a deficiency in G-6-P.D. were consistently found to have reduced GSH stability (Tables II through V). The preincubation concentrations of GSH were lower, on the average, in erythrocytes deficient in G-6-P.D. than in normal red blood cells. There was, however, a considerable overlap in GSH content between these two groups (Table VI).

The results of the GSH stability test expressed as "per cent of GSH protected" were compared to levels of G-6-P.D. (Figure 1). In subjects beyond the first 55 hours of life there was a significant positive correlation (correlation coefficient equals \pm 0.79 , p equals $<0.001$ ) between erythrocyte GSH stability and G-6-P.D. concentrations. This value was based on 123 subjects studied.

TABLE VI

Comparison of erythrocyte concentrations of reduced glutathione and glucose-6-phosphate dehydrogenase

\begin{tabular}{lccc}
\hline \hline \multicolumn{1}{c}{ Subject } & $\begin{array}{c}\text { No. of } \\
\text { subjects }\end{array}$ & $\begin{array}{c}\text { GSH } \\
\text { Mean } \pm \text { S.D. }\end{array}$ & $\begin{array}{c}\text { G-6-P.D. } \\
\text { Mean } \pm \text { S.D. }\end{array}$ \\
\hline Normal enzyme & & $m g . / G m . H g b$. & $\Delta O . D . / G m . H g b . / m i n$. \\
$\quad$ Adults & 76 & $1.47 \pm 0.37$ & $16.2 \pm 2.2$ \\
$\quad \begin{array}{l}\text { Newborns } \\
\text { Deficient enzyme } \\
\text { (all ages) }\end{array}$ & 38 & $1.62 \pm 0.26$ & $20.4 \pm 3.3$ \\
\hline
\end{tabular}

The transient instability of GSH in red blood cells of infants described by Zinkham and Childs (17) was confirmed for normal babies under 55 hours of age. The GSH remaining after incubation of erythrocytes of new borns with acetylphenylhydrazine was 17 to 50 per cent of the preincubation value compared to the normal adult range of 75 to 100 per cent (Table I).

\section{6-Phosphogluconic dehydrogenase and purine nucleoside phosphorylase}

The subjects with decreased levels of erythrocyte G-6-P.D. had concentrations of 6-PG.D. and PNP within the range of normal (Tables I through $\mathrm{V}$ ).

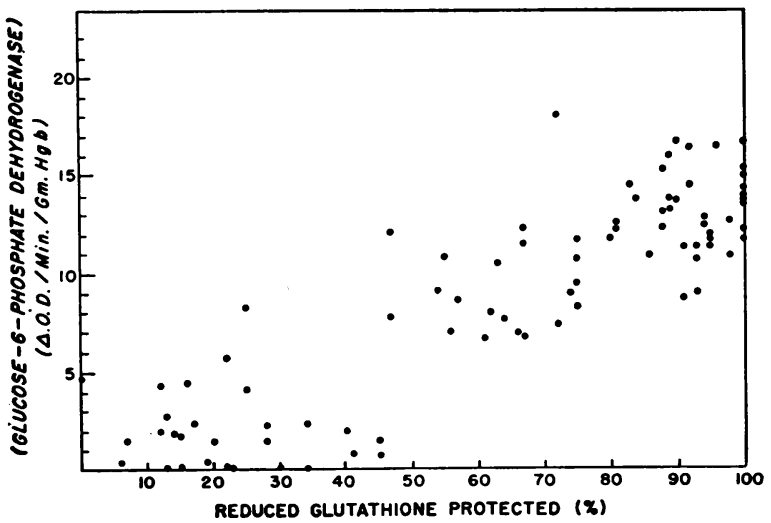

Fig. 1. The Relationship Between Erythrocyte Glucose-6-Phosphate Dehydrogenase (G-6-P.D.) ConCentration and Reduced Glutathione (GSH) StabilITY UPON INCUBATION WITH ACETYLPHENYLHYDRAZINE 


\section{DISCUSSION}

The hereditary basis for a deficiency of G-6P.D. in human red blood cells has been demonstrated in the present study encompassing 18 families, each with at least two members possessing this enzymatic defect. The incidence of decreased erythrocyte G-6-P.D. activity among 103 relatives of 16 propositors was 38 per cent, as compared to an incidence of 4.6 per cent in the control population of 305 subjects (135 infants and 170 adults). ${ }^{3}$ Of the 18 families studied, 15 were Negro families. Among the 152 Negro subjects in the control population, the incidence of this abnormality was 7.2 per cent. Among 153 Caucasian controls the incidence was 1.3 per cent. ${ }^{4}$ The present findings as to the frequency of a deficiency in erythrocyte G-6-P.D. in a group of healthy subjects is in good agreement with earlier observations (5-7).

In 24 of the 52 subjects found to have a deficiency in erythrocyte G-6-P.D., the concentration of the enzyme appeared to be intermediate between the high levels of the controls and the low levels of those who gave a history of drug induced hemolytic anemias. Of these subjects with intermediate concentrations of enzyme, there was a striking predominance of females (23 out of 24 subjects). Females with either intermediate or low levels of erythrocyte G-6-P.D. had male offspring with low or normal, but not intermediate, levels, while among their female offspring, low, normal and intermediate levels occurred. In each of 12 families in which both parents could be tested, one parent had at least a moderate deficiency in red blood cell G-6-P.D. Male children were affected in 14 families, and in each instance the genetic defect was traced to the mother. Among 29 affected male subjects studied, only 4 were parents. The occurrence of the enzymatic defect mainly in male children and female parents, as well as the failure to trace the genetic defect in the male children to their fathers, suggest that sex linkage is the mode of inheritance. These findings are in accord with Childs and associates' (26) as well as Browne's (18) conclusions based on GSH stability studies. Childs has further suggested that the

4 Chi square test revealed that the incidence of erythrocyte G-6-P.D. deficiency in the control group and in the relatives of propositors differed significantly $(\mathrm{p} \ll 0.001)$. heterozygous and homozygous states may possibly be expressed in the intermediate and low levels of GSH stability (26). The present data, based on enzyme levels, suggest that such is not the case. ${ }^{5}$ Although the size of this study does not permit a definite conclusion, the data best fit the postulate that the phenotypic expression of the gene is modified in the female.

Determination of the stability of erythrocyte GSH upon incubation with acetylphenylhydrazine yielded values that indicated a high degree of stability in erythrocytes with normal concentrations of G-6-P.D., markedly decreased stability in erythrocytes with moderate decrease in G-6-P.D. activity. This positive correlation between the level of erythrocyte G-6-P.D. and the stability of GSH is in accord with the previous evidence that erythrocyte GSH metabolism is related to the activity of G-6-P.D. (see reactions 1 through 4). The finding that an infant under three days of age is unable to protect GSH despite high levels of G-6-P.D. is unexplained. It has been shown (19) that the level of erythrocyte G-6-P.D. is very high at birth and does not decrease to within the range of adult levels for approximately 35 weeks. Glutathione reductase activity has been measured in erythrocytes of newborns and was found not to be diminished (20). Whatever the explanation, the fact that erythrocytes of newborn infants may have decreased GSH stability despite high levels of G-6-P.D. precludes the use of GSH studies as a screening test for hereditary erythrocyte drug sensitivity in young infants.

The identifications of G-6-P.D. as an important defect in drug sensitive erythrocytes may explain a curious clinical aspect of the drug induced hemolytic anemias, namely their tendency to be selflimited even in the face of continued ingestion of the agent (2). It has been demonstrated that older erythrocytes have an increased sensitivity to primaquine (21). Recent studies in normal persons $(22,23)$ have shown that the concentration of G-6-P.D. is high in young erythrocytes and decreases markedly with the in vivo aging of these cells. Similar studies in subjects with erythrocyte G-6-P.D. deficiency have revealed that the level

5 In one family a mother with a very low level of G-6-P.D. produced four normal sons. In another mating, a normal father and intermediate mother produced a daughter who was low. 
of this enzyme, while not being comparable to normal values, is higher in young compared with old red cells (24). Thus, drug induced hemolysis may cease when the older cells, deficient in G-6P.D., have been destroyed. The remaining red blood cell population would be composed of young cells, with higher levels of G-6-P.D., and hence, relatively resistant to hemolysis.

In this study the deficiency in G-6-P.D. was encountered in erythrocytes of individuals who never had hemolytic anemia as well as individuals with documented drug induced episodes of hemolysis. $^{6}$ It is not clear by what mechanism a deficiency in G-6-P.D. results in erythrocytes being sensitive to hemolysis following ingestion of certain drugs. Nevertheless, it would seem reasonable to conclude that individuals with a deficiency in erythrocyte G-6-P.D. may be expected to be susceptible to hemolysis following ingestion of such agents as naphthalene, primaquine, nitrofurantoin and fava beans. Accordingly, assay of erythrocyte G-6-P.D. may provide a ready means of detecting such individuals prior to their exposure to drugs potentially harmful to them.

\section{SUMMARY}

1. Erythrocyte glucose-6-phosphate dehydrogenase deficiency has been encountered in 4.6 per cent of an unselected population of 305 subjects. This enzymatic defect was more common among healthy Negroes (7.2 per cent) than among healthy Caucasians (1.3 per cent).

2. Erythrocyte glucose-6-phosphate dehydrogenase deficiency was detected in individuals with a history of hemolytic anemia due to naphthalene ingestion and fava bean consumption, and of undetermined cause.

3. A decrease in erythrocyte reduced glutathione stability upon incubation with acetylphenylhydrazine was consistently associated with a deficiency in glucose-6-phosphate dehydrogenase, except in very young infants. Infants under 55 hours of age demonstrated striking instability in reduced glutathione despite high levels of glucose6-phosphate dehydrogenase activity.

${ }^{6}$ During the preparation of this manuscript the finding of erythrocyte G-6-P.D. deficiency in erythrocytes of individuals who never had hemolytic anemia has also been reported by Schrier, Kellermeyer, Carson, and Alving (25) and is cited by Childs and co-workers (26).
4. The present data establish an hereditary basis for the deficiency of erythrocyte glucose-6phosphate dehydrogenase. They strongly suggest that the gene is sex-linked and also sex-modified.

\section{ACKNOWLEDGMENTS}

The authors are indebted to the following physicians who kindly provided subjects for this study: Major Frederick C. Biehusen and his staff of Letterman Army Hospital; Dr. William Cregar, Department of Medicine, Stanford University School of Medicine; and Dr. James Wolf, Department of Pediatrics, College of Physicians and Surgeons, Columbia University. The authors gratefully acknowledge the technical assistance of Mrs. Judith Beasley and Mr. Stanley Goldstein. Discussions with Doctors R. Ceppellini and C. Stern were very helpful in the genetic interpretation of the present data.

\section{REFERENCES}

1. Beutler, E., Dern, R. J., Flanagan, C. L., and Alving, A. S. The hemolytic effect of primaquine. VII. Biochemical studies of drug-sensitive erythrocytes. J. Lab. clin. Med. 1955, 45, 286.

2. Dern, R. J., Beutler, E., and Alving, A. S. The hemolytic effect of primaquine. II. The natural course of the hemolytic anemia and the mechanism of its self-limited character. J. Lab. clin. Med. 1954, 44, 171.

3. Zinkham, W. H., and Childs, B. Effect of naphthalene derivatives on glutathione metabolism of erythrocytes from patients with naphthalene hemolytic anemia (abstract). J. clin. Invest. 1957, 36, 938.

4. Szeinberg, A., Sheba, C., Hirshorn, N., and Bodonyi, E. Studies on erythrocytes in cases with past history of favism and drug-induced acute hemolytic anemia. Blood 1957, 12, 603.

5. Kimbro, E. I., Jr. The mechanism of hemolytic anemia induced by nitrofurantoin (Furadantin). Bull. Johns Hopk. Hosp. 1957, 101, 118.

6. Beutler, E. The glutathione instability of drug-sensitive red cells. J. Lab. clin. Med. 1957, 49, 84.

7. Hockwald, R. S., Arnold, J., Clayman, C. B., and Alving, A. S. Status of primaquine. 4. Toxicity of primaquine in Negroes. J. Amer. med. Ass. 1952, 149, 1568.

8. Rall, T. W., and Lehninger, A. L. Glutathione reductase of animal tisues. J. biol. Chem. 1952, 194, 119.

9. Marks, P. A. A newer pathway of carbohydrate metabolism; the pentose phosphate pathway. Diabetes 1956, 5, 276.

10. Carson, P. E., Flanagan, C. L., Ickes, C. E., and Alving, A. S. Enzymatic deficiency in primaquinesensitive erythrocytes. Science 1956, 124, 484.

11. Waller, H. D., Löhr, G. W., and Tabatabai, M. Hämolyse und Fehlen von Glucose-6-phosphatdehydrogenase in roten Blutzellen (Eine Fermen- 
tanomalie des Erythrocyten). Klin. Wschr. 1957, 35, 1022.

12. Marks, P. A. Red cell glucose-6-phosphate and 6 phosphogluconic dehydrogenases and nucleoside phosphorylase. Science, 1958, 127, 1338.

13. Horecker, B. L., and Smyrniotis, P. Z. Phosphogluconic acid dehydrogenase from yeast. J. biol. Chem. 1951, 193, 371.

14. Kornberg, A., and Horecker, B. L. Glucose-6-phosphate dehydrogenase in Methods in Enzymology, S. P. Colowick and N. O. Kaplan, Eds. New York, Academic Press Inc., 1955, vol. 1, p. 323.

15. Price, V. E., Otey, M. C., and Plesner, P. Preparation of nucleoside phosphorylase from calf spleen in Methods in Enzymology, S. P. Colowick and N. O. Kaplan, Eds. New York, Academic Press Inc., 1955, vol. 2, p. 448.

16. Grunert, R. R., and Phillips, P. H. A modification of the nitroprusside method of analysis for glutathione. Arch. Biochem. 1951, 30, 217.

17. Zinkham, W. H., and Childs, B. Effect of vitamin $\mathrm{K}$ and naphthalene metabolites on glutathione metabolism of erythrocytes from normal newborns and patients with naphthalene hemolytic anemia. J. Dis. Child. 1957, 94, 420.

18. Browne, E. A. The inheritance of an intrinsic abnormality of the red blood cell predisposing to drug induced hemolytic anemia. Bull. Johns Hopk. Hosp. 1957, 101, 115.

19. Gross, R. T., and Hurwitz, R. E. A comparative study of the glycolytic and pentose phosphate pathways in red blood cells of newborns, prematures, infants, and children. J. Dis. Child. 1957, 94, 487.

20. Gross, R. T., and Hurwitz, R. E. Unpublished data.

21. Beutler, E., Dern, R. J., and Alving, A. S. The hemolytic effect of primaquine. IV. The relationship of cell age to hemolysis. J. Lab. clin. Med. 1954, 44, 439.

22. Marks, P. A. A relationship between human erythrocyte aging in vivo and the activities of glucose-6phosphate and 6-phosphogluconic dehydrogenases (abstract). J. clin. Invest. 1957, 36, 913.

23. Marks, P. A., Johnson, A. B., and Hirschberg, E. Effect of age on the enzyme activity in erythrocytes. Proc. nat. Acad. Sci. (Wash.). In press.

24. Gross, R. T., and Marks, P. A. Unpublished data.

25. Schrier, S., Kellermeyer, R., Carson, P., and Alving, A. S. A second enzyme abnormality in primaquine sensitive erythrocytes. J. Lab. clin. Med. 1957, 50, 951.

26. Childs, B., Zinkham, W. H., Browne, E. A., Kimbro, E. L., and Torbert, J. V. A genetic study of a defect in glutathione metabolism of the erythrocyte. Bull. Johns Hopk. Hosp. 1958, 102, 21. 